\title{
Hardware in the loop simulation of objects internal structures inhomogeneities in multi-angle electrical impedance tomography
}

\author{
Grayr Aleksanyan ${ }^{1, *}$, Nikolay Gorbatenko ${ }^{1}$, Artem Kucher ${ }^{1}$, and Ivan Shcherbakov ${ }^{1}$ \\ ${ }^{1}$ SRSPU (NPI), Department of Information and Measuring Systems and Technologies, 346428 Novocherkassk, Russia
}

\begin{abstract}
The present work is devoted to the development of an algorithm for automatic analysis of images as the results of biological object internal structures conductivity field distribution reconstruction for the automated generation of not only qualitative but also quantitative estimates of inhomogeneities in the tasks of multi-angle multi-frequency electrical impedance tomography. The paper describes the modeling of biological object internal structures inhomogeneities of a given size as a way of obtaining initial data for approbation of the algorithm and its further debugging and optimization. In the work an interactive analysis of the images obtained as a result of modeling is carried out, the results of its work are compared with the results of the developed algorithm of automatic analysis. Based on the analysis, conclusions are drawn about the applicability of the developed algorithm in the problems of multi-angle multi-frequency electrical impedance tomography.
\end{abstract}

\section{Introduction}

Electrical impedance tomography (EIT) is a non invasive effective method to reconstruct and visualize the conductivity distribution in biological objects from the results of electrical measurements on its surface [113]. In the general case, the investigated object (IO) is connected to one or more sources of high frequency electrical current through electrodes placed on its surface, and measured the voltages at the electrodes (where $\mathrm{i}$ is the number pair of injecting electrodes, $\mathrm{j}$ is the number of measuring electrode) [14]. To convert measurement information from the surface of IO in the conductivity distribution in the internal structures of the object is in process of reconstruction, which can be divided into static (absolute), and dynamic (differential). $[14,15]$

Absolute (static) reconstruction is image reconstruction, which uses one dataset for one point in time and returns absolute value of the conductivity in the object. [1,2]

Differential (dynamic) reconstruction is reconstruction, which uses two sets of data at two points in time and returns change of conductivity in the object between the start and end time. [14,15]

When conducting multi-angle multi-frequency electrical impedance tomography (MMEIT), as in any other medical imaging technique, an important role is played by the analysis of the images. Automatic (machine) analysis of images, in contrast to the interactive, allows to obtain quantitative characteristics of the studied objects, and can be used in computer systems of diagnostics, and, what is the most important thing, in decision-making systems.
The purpose of this paper is to develop algorithms for analyzing the results of the reconstruction of conductivity of the internal structures in the task MAIT and debug to re-the results of hardware-software modelling of inhomogeneity of the internal structures of a biological object of a given size.

\section{Materials and Methods}

\subsection{Experimental design}

For modeling inhomogeneity and internal structures of biological object in the phantom [16] (container with polyethylene non-conductive walls with 16 electrodes located along the perimeter) positioned a cylinder of diameter d equal to 13,18 and $27 \mathrm{~mm}$ non-conductive (polyethylene polymer) and conductive (stainless steel) material was placed. Cylinders that simulating inhomogeneity, were placed at fixed distances from the electrode $1(20,40$, and $80 \mathrm{~mm})$ according to a printed target, glued to the bottom of the phantom, as shown in figure 1(a). Figure 1(b-f) shows described above variety of inhomogeneities size and position in the phantom.

To simulate BO internal structure, $0,454 \mathrm{~kg}$ of chicken leg quarter minced in industrial grinder (to avoid metal inclusions that may affect on resulting impedance) with temperature of $4.2^{\circ} \mathrm{C}$ was used, at an ambient temperature of $24.8^{\circ} \mathrm{C}$, atmospheric pressure of $754 \mathrm{~mm}$ $\mathrm{Hg}$; and air humidity of $43 \%$.

To conduct hardware and software simulation used the layout of the hardware-software complex electrical impedance imaging [17-20]. Potential differences $\Delta \varphi$ between all neighboring electrode pairs at a frequency of

\footnotetext{
$\bar{*}$ Corresponding author: graer@yandex.ru
} 
injecting current $\mathrm{f}_{\mathrm{I}}=50 \mathrm{kHz}$ and the amplitude of $\mathrm{I}_{\mathrm{M}}=5$ $\mathrm{mA}$ were measured. These injected current parameters are chosen because of their proven safety for biological object [17], as the simulation of inhomogeneity in biological object is carried out.

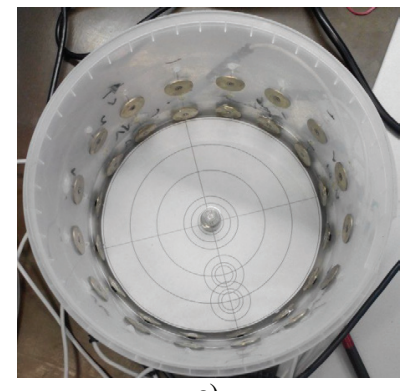

a)

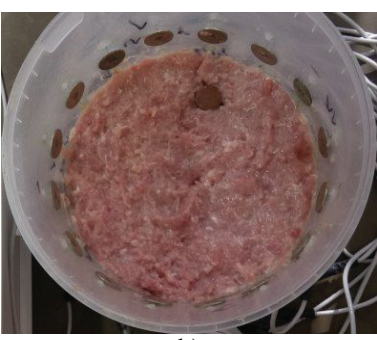

b)

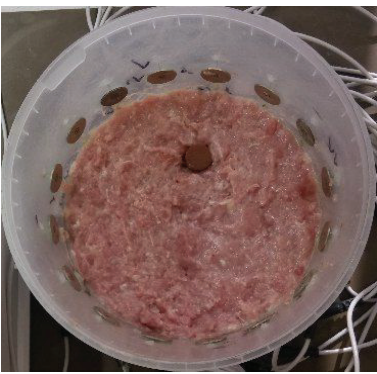

d)

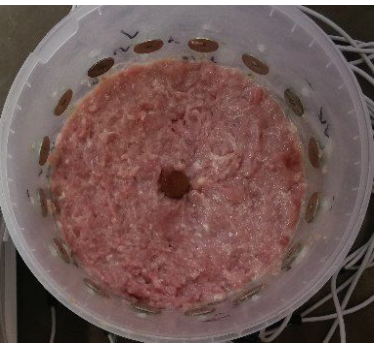

f)

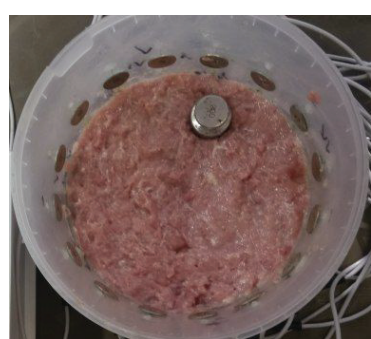

c)

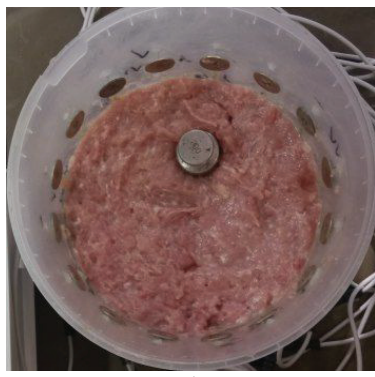

e)

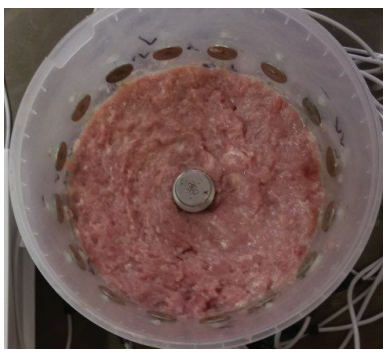

g)
Fig. 1. a) printed target mounted in a bottom of a phantom for correct positioning; b) a non-conductive object with a diameter of $18 \mathrm{~mm}$ at a distance of $20 \mathrm{~mm}$ from the electrode; c) a conductive object with a diameter of $27 \mathrm{~mm}$ at a distance of 20 $\mathrm{mm}$ from the electrode; d) a non-conductive object with a diameter of $18 \mathrm{~mm}$ at a distance of $40 \mathrm{~mm}$ from the electrode; e) a conductive object with a diameter of $27 \mathrm{~mm}$ at a distance of $40 \mathrm{~mm}$ from the electrode; f) a non-conductive object with a diameter of $18 \mathrm{~mm}$ at a distance of $80 \mathrm{~mm}$ from the electrode; g) a conductive object with a diameter of $27 \mathrm{~mm}$ at a distance of $80 \mathrm{~mm}$ from the electrode.

Eleven repeated experiments carried out for each value of the diameter inhomogeneity and the distance between the electrode and center of inhomogeneity and type of inhomoheneity with total count of 198 measurements. As a result, the measurements taken are average values of the above parameters obtained during repeated experiments to reduce measurement inaccuracy. For each successful experiences with the layout of hardware and software, electrical impedance tomography reconstructed image of the matrix with the selected color map each numerical value of the matrix element (figure 2 (a-d)), and implemented interactive (manual visual computer-aided inspection) of the resulting image for later comparison with the results of the developed algorithm.

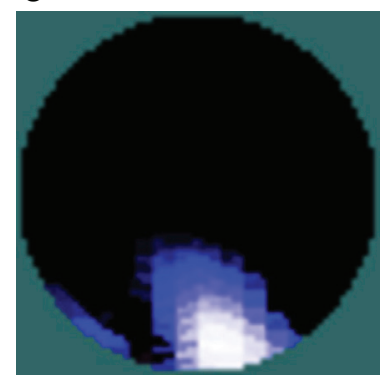

a)

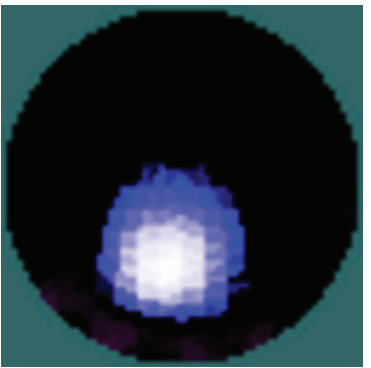

c)

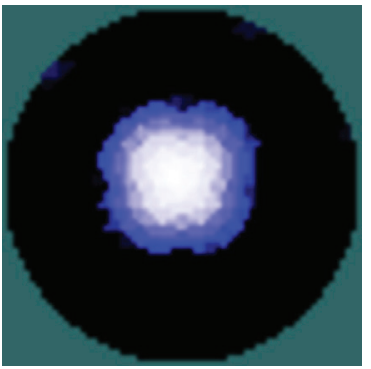

e)

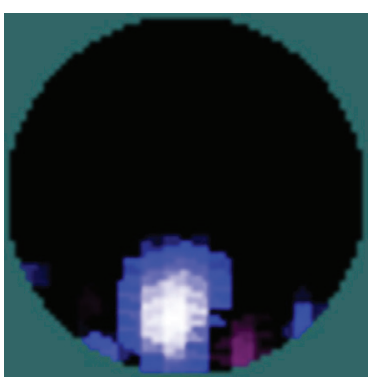

b)

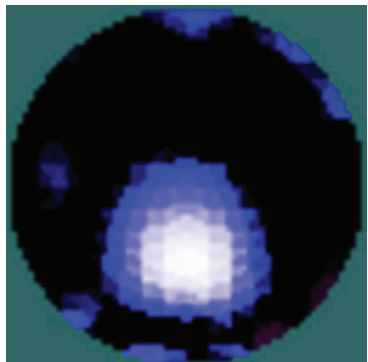

d)

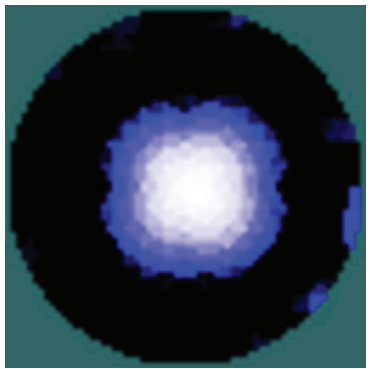

f)
Fig. 2. a) reconstruction: a non-conductive object with a diameter of $18 \mathrm{~mm}$ at a distance of $20 \mathrm{~mm}$ from the electrode; b) reconstruction: a conductive object with a diameter of $27 \mathrm{~mm}$ at a distance of $20 \mathrm{~mm}$ from the electrode; c) reconstruction: a non-conductive object with a diameter of $18 \mathrm{~mm}$ at a distance of $40 \mathrm{~mm}$ from the electrode; d) reconstruction: a conductive object with a diameter of $27 \mathrm{~mm}$ at a distance of $40 \mathrm{~mm}$ from the electrode; e) reconstruction: a non-conductive object with a diameter of $18 \mathrm{~mm}$ at a distance of $80 \mathrm{~mm}$ from the electrode; $\mathrm{f}$ ) reconstruction: a conductive object with a diameter of $27 \mathrm{~mm}$ at a distance of $80 \mathrm{~mm}$ from the electrode.

To carry out interactive analysis, a free and open-source raster graphics editor (GNU General Public License (GNU GPL v3) GIMP was used [21]. Any other available graphics editor with "measure tape" function can be used. The "measure tape" function allows to recognize the distances in pixels between the points of investigated image. Information about the size of the heterogeneity (showed white on fig.2) and the distance from its center to the electrode is obtained by clicking 
and holding the mouse button down, the number of pixels between the click point and the current position of the mouse pointer is determined [22]. The inaccuracy of this type of measurement is determined by the resolution of the image (as a result of the resolution of the reconstruction algorithm used in the EIT tasks) and the width and contrast of the region of the inhomogeneity boundary (showed blue on fig.2): the lower the contrast of this region, the worse the eye distinguishes the position of the boundary.

Since the resolution of the reconstructed image in pixels and the diameter of the phantom in millimeters are known, the size and position of the inhomogeneities can also be calculated from the pixels into metric units (millimeters). Measured data on the size of the inhomogeneity and the distance from its center to the electrode and their measurement errors are recorded for each repeated experiment and averaged.

The block diagram of the algorithm of automatic analysis of investigated object internal structures inhomogeneity presented in fig. 3 .

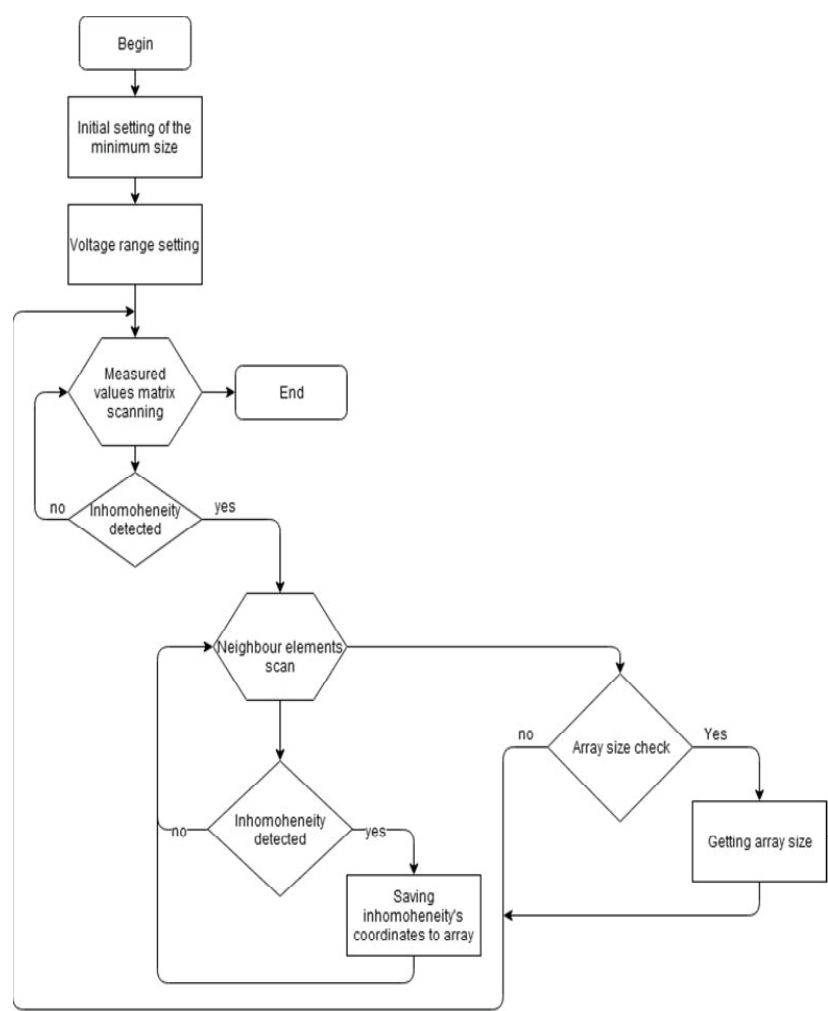

Fig. 3. Block diagram of the developed algorithm for automatic analyzing inhomogeneities of internal structures of the IO.

The principle of its work consists in: the initial setting of the minimum size threshold and voltage ranges (correlated with impedance in term of current source using[20]) of inhomogeneity.

Then the image is scanned as a matrix of voltage values (which are correlating with impedance values) in every pixel of reconstructed image to search for values that match the range of impedances. Upon detection of the first element of inhomogeneity, the values of neighboring elements in the matrix are also tested for inclusion in the range of impedances. If the neighboring elements are also parts of inhomogeneity, the array for storing the coordinates of the positions of the elements of inhomogeneity is created. After all the neighboring elements are tested the size of detected inhomogeneity is determined. If the size of the detected inhomogeneity exceeds a predetermined minimum size, the generated array is stored in memory and the algorithm continues scanning the matrix until find s a new inhomogeneity or finishes their work. The results of the algorithm which also calculated automatically from pixels of reconstructed image to millimeters are displayed in userfriendly form. The inaccuracy of this type of measurement is determined by the resolution of the image too (as a result of the resolution of the reconstruction algorithm used in the EIT tasks), but error caused by width and contrast of the region of the inhomogeneity boundary can be eliminated in this case by correct potential difference (voltage) range settings.

\section{Results}

Table 1 presents the results of the study.

Table 1. Results of the study.

\begin{tabular}{|c|c|c|c|c|c|c|}
\hline \multirow[t]{2}{*}{$\begin{array}{l}\text { Obj. } \\
\text { type }\end{array}$} & \multicolumn{2}{|c|}{$\begin{array}{l}\text { Physical } \\
\text { dimensions of } \\
\text { inhomogeneity }\end{array}$} & \multicolumn{2}{|c|}{$\begin{array}{l}\text { The results of } \\
\text { the interactive } \\
\text { analysis }\end{array}$} & \multicolumn{2}{|c|}{$\begin{array}{lr}\text { The results } \\
\text { obtained by } \\
\text { developed } \\
\text { algorithm }\end{array}$} \\
\hline & $d, m m$ & $l, \mathrm{~mm}$ & $d, m m$ & $l, \mathrm{~mm}$ & $d, m m$ & $l, \mathrm{~mm}$ \\
\hline \multirow{9}{*}{ 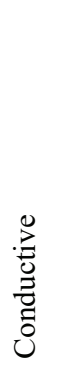 } & $13 \pm 0,5$ & $20 \pm 0,5$ & $19 \pm 4$ & $17 \pm 5$ & $15 \pm 2,5$ & $19 \pm 2,5$ \\
\hline & $13 \pm 0,5$ & $40 \pm 0,5$ & $20 \pm 4$ & $25 \pm 7$ & $11 \pm 2,5$ & $32 \pm 2,5$ \\
\hline & $13 \pm 0,5$ & $80 \pm 0,5$ & $25 \pm 5$ & $65 \pm 15$ & $17 \pm 2,5$ & $70 \pm 2,5$ \\
\hline & $18 \pm 0,5$ & $20 \pm 0,5$ & $28 \pm 5$ & $12 \pm 10$ & $22 \pm 2,5$ & $20 \pm 2,5$ \\
\hline & $18 \pm 0,5$ & $40 \pm 0,5$ & $26 \pm 8$ & $22 \pm 15$ & $23 \pm 2,5$ & $35 \pm 2,5$ \\
\hline & $18 \pm 0,5$ & $80 \pm 0,5$ & $28 \pm 12$ & $76 \pm 5$ & $16 \pm 2,5$ & $77 \pm 2,5$ \\
\hline & $27 \pm 0,5$ & $20 \pm 0,5$ & $24 \pm 14$ & $10 \pm 8$ & $25 \pm 2,5$ & $20 \pm 2,5$ \\
\hline & $27 \pm 0,5$ & $40 \pm 0,5$ & $33 \pm 5$ & $34 \pm 4$ & $29 \pm 2,5$ & $33 \pm 2,5$ \\
\hline & $27 \pm 0,5$ & $80 \pm 0,5$ & $37 \pm 4$ & $94 \pm 10$ & $25 \pm 2,5$ & $90 \pm 2,5$ \\
\hline \multirow{9}{*}{ 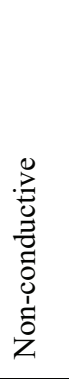 } & $13 \pm 0,5$ & $20 \pm 0,5$ & $19 \pm 4$ & $17 \pm 5$ & $15 \pm 2,5$ & $19 \pm 2,5$ \\
\hline & $13 \pm 0,5$ & $40 \pm 0,5$ & $20 \pm 4$ & $24 \pm 8$ & $14 \pm 2,5$ & $32 \pm 2,5$ \\
\hline & $13 \pm 0,5$ & $80 \pm 0,5$ & $19 \pm 5$ & $56 \pm 15$ & $15 \pm 2,5$ & $68 \pm 2,5$ \\
\hline & $18 \pm 0,5$ & $20 \pm 0,5$ & $28 \pm 5$ & $12 \pm 9$ & $22 \pm 2,5$ & $20 \pm 2,5$ \\
\hline & $18 \pm 0,5$ & $40 \pm 0,5$ & $25 \pm 8$ & $22 \pm 12$ & $23 \pm 2,5$ & $35 \pm 2,5$ \\
\hline & $18 \pm 0,5$ & $80 \pm 0,5$ & $28 \pm 12$ & $76 \pm 5$ & $16 \pm 2,5$ & $77 \pm 2,5$ \\
\hline & $27 \pm 0,5$ & $20 \pm 0,5$ & $23 \pm 13$ & $8 \pm 6$ & $24 \pm 2,5$ & $19 \pm 2,5$ \\
\hline & $27 \pm 0,5$ & $40 \pm 0,5$ & $32 \pm 6$ & $34 \pm 4$ & $28 \pm 2,5$ & $32 \pm 2,5$ \\
\hline & $27 \pm 0,5$ & $80 \pm 0,5$ & $39 \pm 5$ & $89 \pm 8$ & $23 \pm 2,5$ & $86 \pm 2,5$ \\
\hline
\end{tabular}

Conducted analysis of the data presented in table 1 shows that the spread of the values of the inhomogeneity parameters (diameter and position) measured in an interactive (manual visual, computer-aided) way is greater in all cases than the spread of the values obtained as a result of physical measurements, and in most cases exceeds the spread of the values obtained with the developed algorithm, what can be explained by the impossibility of registering with the human eye individual shades of the pixel colors in the conditions of fuzzy boundaries of the object on the reconstructed image. Also worth noting is the lower accuracy of the results of the developed algorithm compared to the data 
obtained as a result of physical measurements of real objects, not least due to the relatively low accuracy of the results of the reconstruction algorithms (low resolution), that is input data for the image analysis algorithm.

\section{Conclusion}

Hardware and software modeling of inhomogeneities internal structures of objects using the layout of hardware and software electrical impedance tomography was conducted. The algorithm of automatic analysis of inhomogeneity of the internal structures of the object was developed.

A comparison of measured information consisting of the parameters of the inhomogeneity (the diameter and distance to electrode) and measurement errors was conducted on data:

- obtained as the result of the physical measurements of real objects (diameter of phantom, inhomogeneity cylinders samples);

- obtained as the result of the interactive (manual and visual, computer-aided, with graphics editor) analysis of the reconstructed image;

- obtained as the result of the developed algorithm of automatic analysis of inhomogeneity of the internal structures in the object.

The analysis of the comparison shows higher accuracy of the measurement of inhomogeneity with developed algorithm comparing to results of interactive image analysis, and higher efficiency of its use in problems of multi-angle multi-frequency electrical impedance tomography as a tool for the automatic analysis of the inner structures of IO. At the same time, also worth noting is the lower accuracy of the results of developed algorithm compared to the data obtained as a result of physical measurements of real objects, not least due to the relatively low accuracy of the results of the reconstruction algorithms that is input data for the image analysis algorithm.

Several conclusions can be conducted from above:

- developed algorithm has inaccuracy of inhomogeneity positioning due to inaccuracy of reconstruction algorithm;

- -developed algorithm has less factors affecting accuracy of measurement comparing to traditional interactive analysis (even computer-aided) which is widely used in clinical practice;

- developed algorithm of automatic analysis of investigated object internal structures can be used in tasks of multi-angle multi-frequency electrical impedance tomography;

- developed algorithm can be used in a decisionmaking systems with some accuracy limitations (which is the subject of further research).

Work is performed within the grant of President of Russian Federation for state support of young Russian scientists MK196.2017.8 "Development of theoretical foundations and algorithms for multi-view systems are three-dimensional electrical impedance tomography for non-invasive medical imaging".

\section{References}

1. S.Martin, C.T.M. Choi, Sci. Rep., 7(1),7212 (2017)

2. J. Zhang, B.Yang, , H. Li, X.Dong, , M. Dai, Sci. Rep., 7(1),4608 (2017)

3. N. Eronia, T.Mauri, E. Maffezzini, etc., Annals of Intensive Care, 7(1), 76 (2017)

4. Y. Zhou, X. Li, Measurement: J Int. Meas. Confed., 110, 27-42 (2017)

5. M.G. Crabb, Inverse Prob. in Sci. \& Eng., 25(10), 1397-1422 (2017)

6. M.G. Crabb, Inverse Prob. in Sci. \& Eng., 25(10), X (2017)

7. T.N. Tallman, J.A. Hernandez, NDT and E International, 91, 156-163 (2017)

8. A. Motamed Dashliborun, M. Hamidipour, F. Larachi, AIChE J, 63(9), 4161-4176 (2017)

9. J.S. Martins, C.S. Moura, R.M.F. Vargas, Inverse Prob. in Sci. \& Eng., 1-21 (2017)

10. S. Białka, M. Copik, K. Rybczyk, H. Misiołek, Anaesthesiology Intensive Therapy, 49(3), 222-226 (2017)

11. Y. Liu, Y. Deng, M. Zhang, P. Yu, Y. Li, Meas. Sci. \& Tech., 28(9),095301 (2017)

12. G. González, V. Kolehmainen, A. Seppänen, Comp. \& Math. with Appl., 74(3), 564-576 (2017)

13. Y. Yang, J. Jia, Rev. of Sci. Instrum., 88(8), 085110 (2017)

14. Y.S. Pecker, K.S. Brazovskii, V.Y. Usov, M.P. Plotnikov, O.S. Umansky, Electrical impedance tomography ("NTL" Publishing House, Tomsk, 2004)

15. D.S. Holder Electrical Impedance Tomography. Methods, History and Applications (Institute of Physics Publishing, Bristol and Philadelphia, 2005).

16. G.K. Aleksanyan, N.I. Gorbatenko, A.I. Kucher, Int. J Appl. Eng. Res., 10 (19), 40580-40584 (2015)

17. G.K. Aleksanyan, I.D. Shcherbakov, A.I. Kucher, V.V. Demyanov, J Eng. \& Appl. Sci., 12 (8), 21292137 (2017)

18. G.K. Aleksanyan, N.I. Gorbatenko, A.I. Kucher, Int. J Appl. Eng. Res., 10 (19) (2015)

19. G.K. Aleksanyan, A.I. Kucher, A.D. Tarasov, N.M. Cuong, C.N. Phong, Int. J Soft Comp., 10 (6), 462467 (2015)

20. G.K. Aleksanyan, I.D. Shcherbakov, A.I. Kucher, J Eng. \& Appl. Sci., 12 (3), 587-592 (2017)

21. A. Peck, Beginning GIMP: From Novice to Professional. (Apress Inc, 2006)

22. Carey Bunks. Grokking the Gimp. (New Riders Publishing, 2000) 